\title{
Efecto del ejercicio físico sobre la condición física en adultos con lesión medular: una revisión sistemática
}

\author{
Effect of physical exercise on physical condition in adults with medular injury: a \\ systematic review
}

\author{
Daniel Efrén García-González ${ }^{1}$ D, Carolina Sandoval-Cuellar ${ }^{2}$ (D)
}

Citación: García-González DE, Sandoval-Cuéllar C. Efecto del ejercicio físico sobre la condición física en adultos con lesión medular: una revisión sistemática. Ustasalud 2021;20: 31-42.

Licencia Creative Commons

\section{(c) (1) (\$) $\ominus$} lo tanto, los lectores pueden acceder libremente a los artículos en su formato .pdf, igualmente podrán descargarlos y difundirlos; sin embargo no podrán modificarlos o alterarlos, adicionalmente se debe reconocer la autoría de las personas que figuran en las publicaciones, pero estas no podrán ser comercializadas.

\section{Resumen}

Esta revisión pretende determinar el efecto del ejercicio físico sobre la capacidad física, fuerza-resistencia muscular, composición corporal y rendimiento funcional en adultos con lesión medular. La búsqueda sistemática se hizo en las bases de datos PubMed, Trip Medical Database, Scopus, Web of Science, Registro Cochrane Central de Ensayos Controlados, Scielo y ScienceDirect entre los años 1990-2016. Se identificaron 2906 artículos. El 31\% de los estudios incluidos fueron realizados en Canadá, 46,1\% en Estados Unidos y Australia, 15,3\% en Holanda y 7,6\% en Brasil, el tiempo postoperatorio para intervención con ejercicio físico osciló entre 11,58 meses y 24 años. El 100\% de los estudios reportaron el protocolo de intervención utilizado en términos de longitud, duración, frecuencia e intensidad, el $46 \%$ de los estudios reportó niveles de lesión medular. Los resultados de las intervenciones mediadas a través de ejercicio físico sobre los componentes de la salud física en pacientes con lesión medular son alentadores. Los estudios analizados evidencian los beneficios sobre la capacidad física, fuerza y resistencia muscular, composición corporal y rendimiento funcional en pacientes con lesión medular que favorecen la calidad de vida y el bienestar psicológico de dicha población.

Palabras clave: Traumatismos de la médula espinal, ejercicio, fuerza muscular, composición corporal, entrenamiento de resistencia.

\begin{abstract}
This review aims to determine the effect of physical exercise on physical capacity, muscular strengthendurance, body composition and functional performance in adults with spinal cord injury (SCl). The systematic search was carried out in the databases PubMed, Trip Medical Database, Scopus, Web of Science, Cochrane Central Register of Controlled Trials, Scielo and ScienceDirect between the years 1990-2016. 2906 articles were identified in the consulted databases. $31 \%$ of the included studies were conducted in Canada, $46.1 \%$ in the United States and Australia, $15.3 \%$ in the Netherlands and $7.6 \%$ in Brazil, the postoperative time for intervention with PE ranged between 11.58 months and 24 years. $100 \%$ of the studies reported the intervention protocol used in terms of length, duration, frequency and intensity, $46 \%$ of the studies reported levels of spinal cord injury. The results of the interventions mediated through PE on the components of physical health in patients with spinal cord injury are encouraging. The analyzed studies show the benefits on physical capacity, muscular strength and endurance, body composition and functional performance in patients with spinal cord injury that favor quality of life and the psychological well-being of said population.
\end{abstract}

Keywords: Spinal cord injuries, exercise, muscle strength, body composition, and resistance training.
1 Magíster en Actividad Física y Salud Centro de Estudios para la Medición de la Actividad Física. Escuela de Medicina y Ciencias de la Salud, Universidad del Rosario, Bogotá D.C., Colombia.

2 Magíster en Intervención Integral del Deportista. Centro de Estudios para la Medición de la Actividad Física. Escuela de Medicina y Ciencias de la Salud, Universidad del Rosario, Bogotá D.C., Colombia.

Autor de Correspondencia: Daniel Efrén García-González Correo electrónico: danielegg05@gmail.com.
Recibido para publicación: 8 de septiembre de 2020 Aceptado para publicación: 17 de febrero de 2021 


\section{INTRODUCCIÓN}

La lesión medular (LM) puede definirse como todo proceso patológico (conmoción, contusión, laceración, compresión o sección), de cualquier etiología (traumática y no traumática), que afecta la médula espinal y puede originar alteraciones de la función neurológica por debajo de la lesión: motoras, sensitivas y autonómicas [1]. Se calcula que su incidencia mundial anual oscila entre 40 y 80 casos por millón de habitantes, siendo Estados Unidos uno de los países donde existe mayor número de personas con dicha condición $[2,3]$.

Los traumatismos constituyen la primera causa de LM, presentándose el $60 \%$ de los casos en países desarrollados y cerca del $80 \%$ en países en vía de desarrollo; allí, los traumas son ocasionados por heridas de armas blancas y de fuego, accidentes de tránsito, caídas, zambullidas en aguas poco profundas, accidentes deportivos y laborales [1].

Este tipo de lesión ocurre predominantemente entre los 15 a 35 años con una distribución por sexo (hombre / mujer) de 4:1, el nivel neurológico más afectado suele ser el cervical C5; además, se asocia a menores tasas de escolarización, participación económica y supone un costo importante para quienes las padecen como para la sociedad en su conjunto $[2,4]$.

Los individuos con LM presentan alteraciones en los cuatro componentes de la salud física: capacidad física, fuerza-resistencia muscular, composición corporal y rendimiento funcional, desencadenando complicaciones en salud secundarias, tales como obesidad, enfermedades cardiovasculares, diabetes tipo 2, hipertensión arterial, síndrome metabólico, osteoporosis, dolor crónico e infecciones de la piel y de la vejiga [5].

Dentro de las afecciones características en cada uno de los componentes, la pérdida de la capacidad física se caracteriza por una disminución de la capacidad pulmonar y una alteración del control cardiovascular por la interrupción de la entrada descendente al sistema nervioso autónomo (SNA), generando un efecto significativo en la presión arterial, la frecuencia cardíaca y la regulación de la temperatura. Además, se presenta una serie de cambios en el sistema músculo esquelético, incluyendo la atrofia, que conlleva la disminución de la fuerza muscular y resistencia a la fatiga, sumado al desacondicionamiento físico [6].

A nivel morfológico, los cambios en la composición corporal se producen por la presencia de obesidad, debido a la capacidad reducida de los pacientes con LM de incorporar todos los músculos para la realización de ejercicio físico (EF), lo cual resulta en una pérdida marcada de masa muscular y en un aumento de la adiposidad corporal, haciendo difícil la realización de las actividades cotidianas de manera independiente y autónoma. Sumado a ello, la pérdida marcada de la densidad mineral ósea (DMO) como resultado de cambios neurales, vasculares y hormonales, incrementa el riesgo de osteoporosis y fracturas $[4,6]$.

Entre las estrategias de intervención física, aplicadas en pacientes con LM se incluyen: la Electro Estimulación Funcional (EEF), el entrenamiento aeróbico, entrenamiento en circuitos, la actividad física asistida por robótica, participación en actividades deportivas, programas de rehabilitación con trabajo de peso libre, bandas elásticas, uso de máquinas de levantamiento de peso, ergometría del brazo, entrenamiento de la marcha en cinta rodante con soporte de peso, modificaciones en la dieta, natación y otros deportes, que favorezcan la participación, inclusión social y comunitaria $[4,7$, 8]. Todas estas estrategias están orientadas a mejorar la resistencia aeróbica, la salud cardiovascular, la habilidad y movilidad de las articulaciones, la extensibilidad muscular, evitar la pérdida ósea, reducir el dolor y la espasticidad, mejorando así la calidad de vida (CV) [7].

En la actualidad, no existe evidencia conocida que sistematice los efectos del EF sobre la LM para poder establecer conclusiones significativas de este sobre los componentes de la salud física, principalmente en lesiones agudas, requiriéndose para ello estudios de mayor calidad [9]. Por otra parte, otros autores han reportado evidencia ambigua y de poca calidad con respecto a los efectos del EF sobre la composición corporal y el rendimiento funcional en personas con $\operatorname{LM}[3,10]$.

Con base en lo anterior, se decidió realizar esta revisión sistemática, con el objetivo de evidenciar el 
efecto del EF sobre la capacidad física, fuerza y resistencia muscular, composición corporal y rendimiento funcional en adultos con lesión medular.

\section{MATERIALES Y MÉTODOS}

El estudio responde a una revisión sistemática de la literatura acorde con los lineamientos descritos en la declaración PRISMA $[11,12]$ y en el Manual Cochrane de revisiones sistemáticas de intervenciones, versión 5.1.0 [13].

Dentro de los criterios de inclusión se tuvieron en cuenta ensayos clínicos controlados aleatorizados (ECCA) que fueran publicados desde 1990 hasta 2016, verificando el cumplimiento de los criterios de selección establecidos según la estrategia (PICO), agrupando la búsqueda en los siguientes temas: población $(\mathrm{P})$, intervención (I), grupo control $(\mathrm{C})$ y Outcome o resultado (O). Durante su desarrollo no se aplicaron limitaciones lingüísticas.

Se excluyeron investigaciones realizadas en pacientes menores de 18 años, estudios con experimentación animal, estudios con protocolos de intervención en actividades, como Yoga, Tai-chi, meditación, medicina alternativa, entre otras; investigaciones en las que el diseño no fuera claro o no incluyeran en su modelo un grupo de control, estudios realizados en pacientes que no presenten algún tipo de LM (completa e incompleta), y estudios que únicamente incluyeran intervenciones farmacológicas en los pacientes con LM.

Esta búsqueda sistemática se hizo en las bases de datos de PubMed, Trip Medical Database, Scopus, Web of Science y las bibliotecas electrónicas Scielo y ScienceDirect. Para ello, se utilizaron los operadores lógicos AND, OR y NOT que fueron usados para combinar las palabras clave extraídas de los Descriptores en Ciencias de la Salud (DeCS) y de los Medical Subject Headings (MeSH): muscle strength, breast cancer, quality of life, exercise, resistence training; de esta manera, lograr una selección específica de la literatura.

La búsqueda fue realizada por dos autores cegados e independientes, durante el periodo: diciembre del 2015 hasta julio de 2016, la cual tuvo en cuenta las recomendaciones de Robinson y Dickersin [14].
Los resultados obtenidos describieron las características generales de la población, el tipo de estudio, el tipo de evaluación e intervención, las variables analizadas y los principales resultados encontrados.

\section{Selección de estudios}

La búsqueda y proceso de selección de los artículos se llevó a cabo mediante cuatro fases, las cuales permitieron a los autores seleccionar 13 artículos como muestra el diagrama de flujo (Fig. 1). En la primera fase (identificación), se encontraron 2906 estudios; 2641 fueron eliminados: 1209 textos científicos duplicados, 612 entre metaanálisis, revisiones sistemáticas, estudios descriptivos, estudios en curso, modelos con animales, intervenciones en niños, intervenciones con yoga, taichí o masaje, asunto principal e intervenciones en pacientes sin lesión medular y 820 protocolos de ECCAs. Durante la segunda fase (screening), se prescindió de un total de 13 estudios por no cumplir los criterios de selección propuestos. En la tercera etapa (elegibilidad) se excluyeron 239 manuscritos, estableciendo en la cuarta y última etapa (inclusión) 13 artículos científicos con los cuales se adelantó el presente estudio (Fig. 1). Los resultados de la búsqueda fueron sintetizados en una hoja de cálculo de Microsoft Excel@ 2013.

\section{Extracción de datos}

Un autor de forma independiente (DEGG) extrajo los datos y los registró en una hoja de cálculo electrónica en Microsoft Excel@. Proceso verificado por un segundo autor cegado (CSC) frente a los resultados del primer autor. Los datos extraídos de cada estudio fueron: autores, año de publicación, diseño del estudio, nivel de lesión, clasificación (American Spinal Injury Association) ASIA, tiempo después de la lesión, tamaño de la muestra, características de las intervenciones (intensidad, frecuencia y duración), modalidad (es) de actividad física (AF), edad de los pacientes, instrumento y medidas de resultado.

\section{Evaluación de la calidad metodológica}

Dos autores (DEGG y CSC), de manera independiente, evaluaron la calidad metodológica y el riesgo de sesgo de los estudios incluidos, utilizando la escala PEDro [16], basada en la lista Delphi [13, 17]. Esta escala 
establece a cada ítem una puntuación de 0 a 10 para determinar la calidad metodológica de los ECCA. Cada estudio obtiene una calificación a través de la información disponible, tanto de la versión publicada como de la comunicación con los autores. El presente estudio estableció una puntuación de 5/10 como el valor mínimo para la inclusión de los ensayos en este [18]. Para ello, un autor independiente y capacitado (DEGG) realizó el proceso, a cada ítem se le asignó un Sí cumple (calificado como 1) o un NO cumple (calificado como 0), siendo ponderados con igual importancia (Tabla 1).

Adicionalmente, para el ECCA que no fue encontrado durante el proceso de evaluación de calidad metodológica, un autor independiente y capacitado
(DEGG) hizo la evaluación metodológica del mismo manualmente, bajo los criterios establecidos según PEDro. Para ser incluido en la etapa de extracción de información y análisis de resultados, se estableció que el estudio debería cumplir al menos cinco ítems de la escala PEDro.

En total, los trece estudios incluidos cumplieron con los criterios de inclusión establecidos para la presente revisión; además de los criterios de asignación aleatoria, estimaciones puntuales y variabilidad, que establece la escala PEDro. Sin embargo, ninguno de los estudios incluidos cumple con los criterios de sujetos y terapeutas cegados. Los ECCA [7, 21, 23, 24] informaron presencia de efectos adversos en las intervenciones con AF y EF.
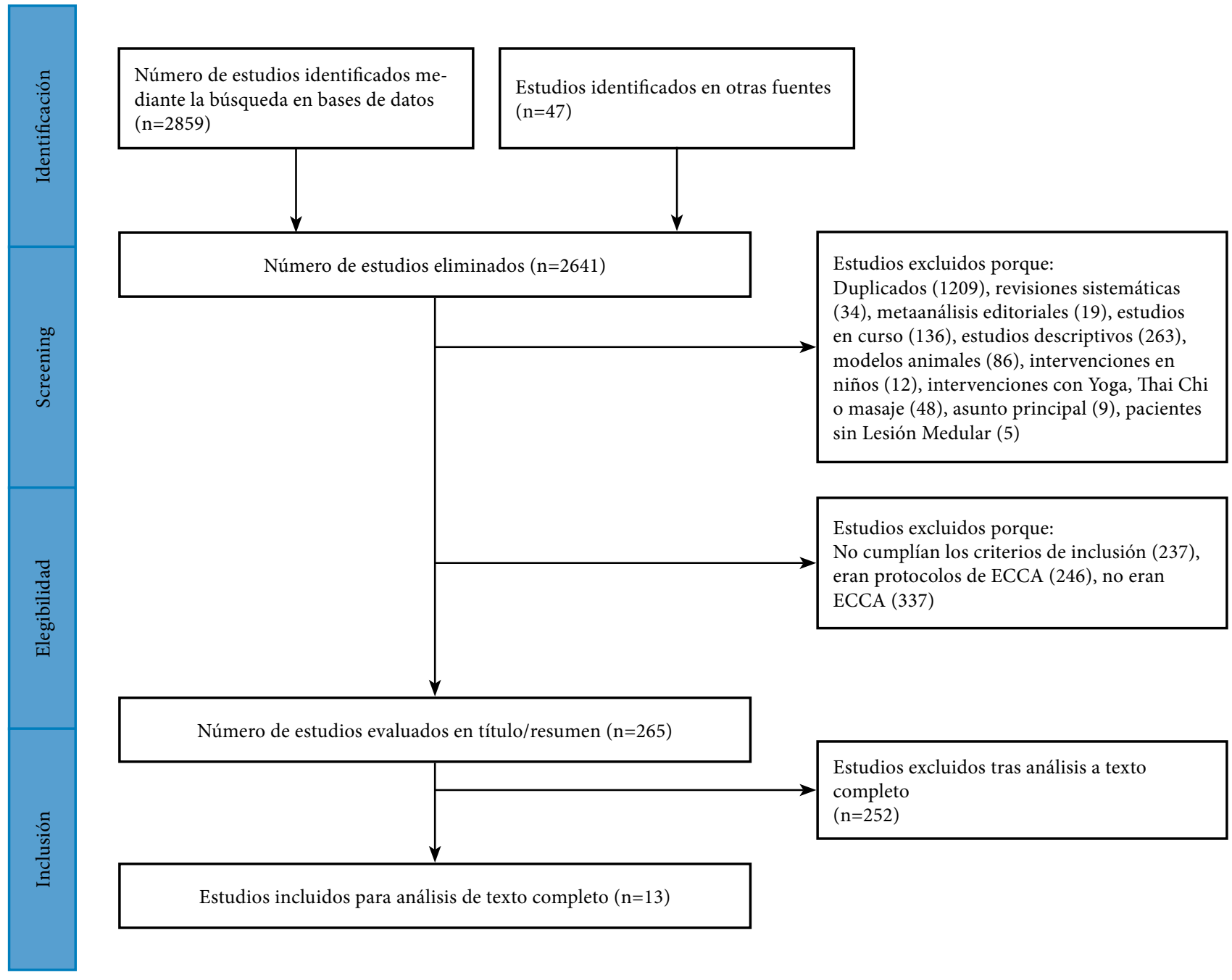

Figura 1. Diagrama de flujo para los métodos de la estrategia de búsqueda de acuerdo con la declaración PRISMA. 


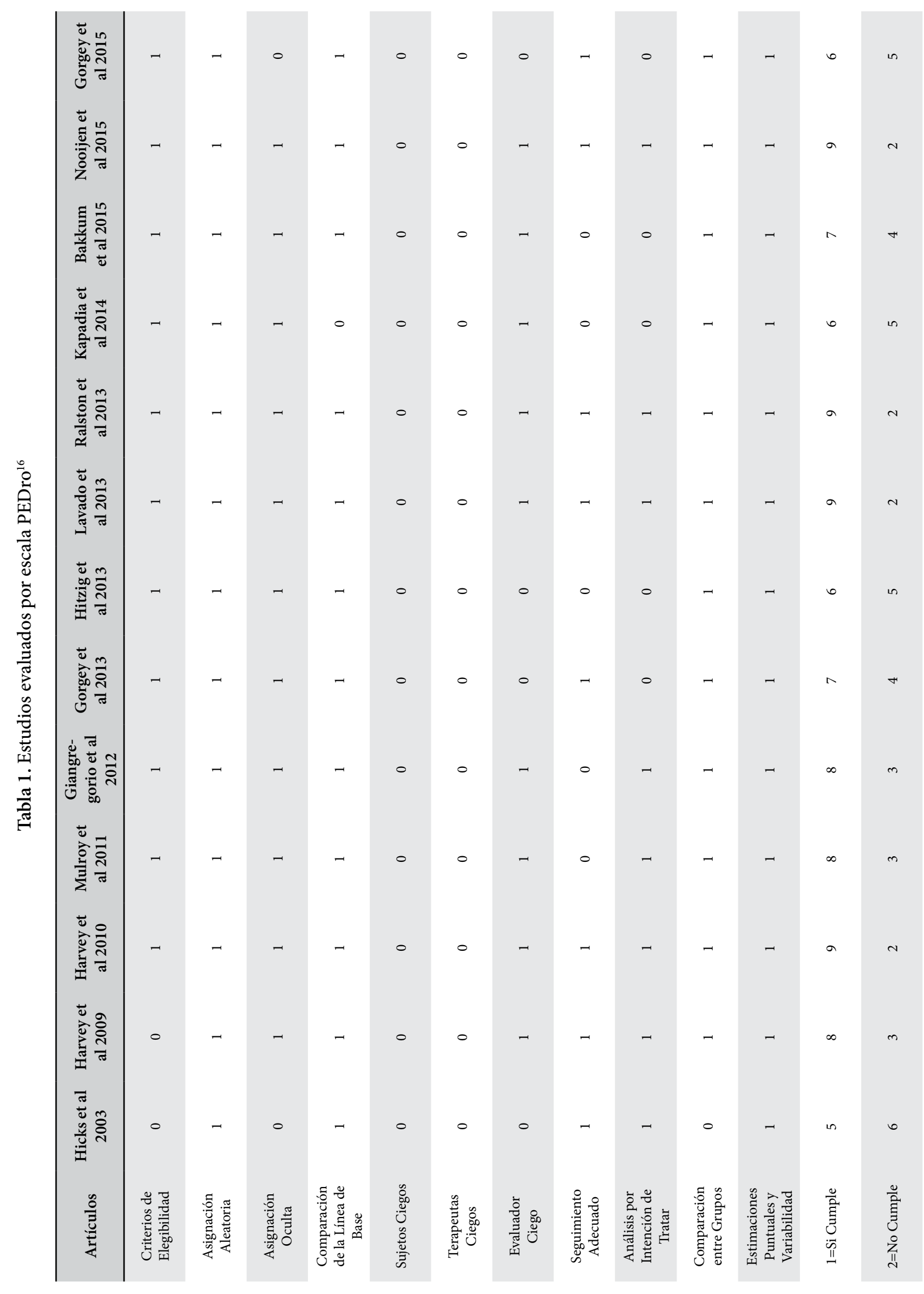




\section{RESULTADOS}

Se identificaron 2906 artículos en las bases de datos consultadas. El $31 \%$ de los estudios incluidos fueron realizados en Canadá, 46,1\% en Estados Unidos y Australia, 15,3\% en Holanda y 7,6\% en Brasil, el tiempo postoperatorio para intervención con EF osciló entre 11,58 meses y los 24 años (Tablas 2 y 3 ). El 100\% de los estudios reportaron el protocolo de intervención utilizado en términos de longitud, duración, frecuencia e intensidad, el $46 \%$ de los estudios reportó niveles de lesión medular (C2-T12-ASIA C y D). El promedio de edad fue 41,43 años y los tamaños de muestra utilizados de 7 hasta 80 sujetos, un 54\% de los estudios incluyó hombres. Estos estudios fueron realizados en población europea, asiática, afrodescendiente, latina, australiana y blancos. Además, las mediciones en los grupos controles se caracterizaron por ser de 8 hasta 16 semanas, con intervenciones en dieta ( $45 \%$ carbohidratos, $30 \%$ grasas y $25 \%$ proteína), acciones educativas, técnicas de relajación, manejo del dolor, videos de anatomía del hombro e instrucciones para acciones de la vida diaria.

Finalmente, todos los estudios incluyeron evaluación de alguno de los componentes de la salud física; sin embargo, difirieron en el tipo de instrumento para la medición.

\section{DISCUSIÓN}

Los resultados de las intervenciones mediadas a través de EF sobre los componentes de la salud física en pacientes con LM son alentadores. Los estudios analizados evidencian los beneficios sobre la capacidad física, fuerza y resistencia muscular, composición corporal y rendimiento funcional en pacientes con LM que favorecen la CV y el bienestar psicológico de dicha población [23, 25, 29].

Los resultados encontrados por Bakkum et al. [27] y Lavado et al. [20], sugieren que el entrenamiento supervisado de ejercicio aeróbico en pacientes con LM a intensidades entre el 65\% hasta el 80\% FCmáx mejoran de manera significativa la capacidad física mediante el incremento del VO2peak y la reducción de la adiposidad visceral; sin embargo, Hicks et al. [29] evidencian que las intervenciones con intensidades entre el 70\% y el 90\% de la FCmáx, con 120 minutos de trabajo por sesión, favorecen el desarrollo de la capacidad física, fuerza y resistencia muscular, CV y bienestar psicológico.

Por otra parte, Harvey et al. [19]; Lavado et al. [20]; Kapadia et al. [26] y Gorgey et al. [30] demostraron que los cambios morfológicos en las estructuras musculares para el desarrollo de la fuerza en miembros inferiores contemplan protocolos con una frecuencia de entrenamiento de 2-3 veces por semana, intensidad moderada a vigorosa ( $65 \%$ al $80 \%$ FC) compuestas por 3 series de $8-10$ repeticiones. Contrario a ellos, Mulroy et al. [23] y Hicks et al. [29] utilizaron protocolos de intervención que favorecieron el desarrollo de la fuerza en miembros superiores a nivel de los músculos bíceps y deltoides, facilitando movimientos como la abducción, elevación escapular, rotación externa y rotación interna del hombro.

Frente a la composición corporal, los estudios de Ralston et al. [21]; Giangregorio et al. [24] y Gorgey et al. [28] coinciden en que las intervenciones físicas mediadas a través de EEF reducen la masa grasa, el peso corporal, el porcentaje graso corporal, el IMC, perímetro o circunferencia abdominal y la grasa en pantorrilla; además que, la EEF favorece el incremento del músculo magro a nivel corporal. Sin embargo, Bakkum et al. [27] obtuvieron efectos sobre la composición corporal en la reducción de la grasa del tronco y androide, a través de intervención con cicloergómetro de brazo.

Los beneficios a nivel del rendimiento funcional fueron observados en su mayoría mediante protocolos diversos, algunos con la implementación de EEF, EEF+Lokomat 70, Robot-Assisted Gait Training y Body Weight-Supported Gait Training, obteniendo beneficios en la velocidad media de marcha, habilidades sobre la silla de ruedas, propulsión en la silla de ruedas y desarrollo de limitaciones en actividades de la vida diaria [10, 22, 23, 34, 35]. Diferente a ellos, Harvey et al. [7] realizaron un programa de intervención mediante el entrenamiento de la flexibilidad, demostrando cambios significativos a nivel de la amplitud del rango articular del hombro y la flexibilidad de los músculos de la espalda y extremidades superiores. 


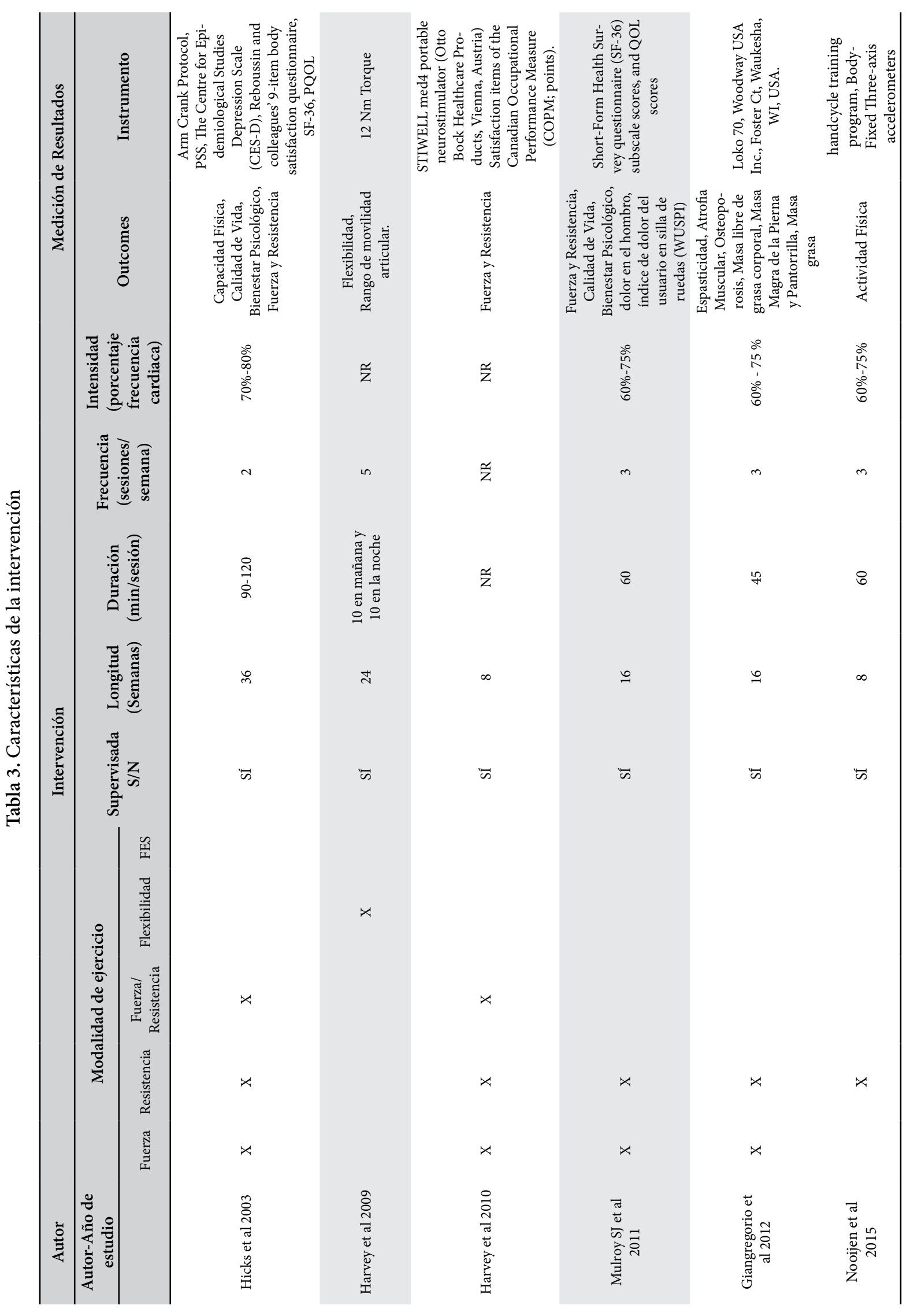




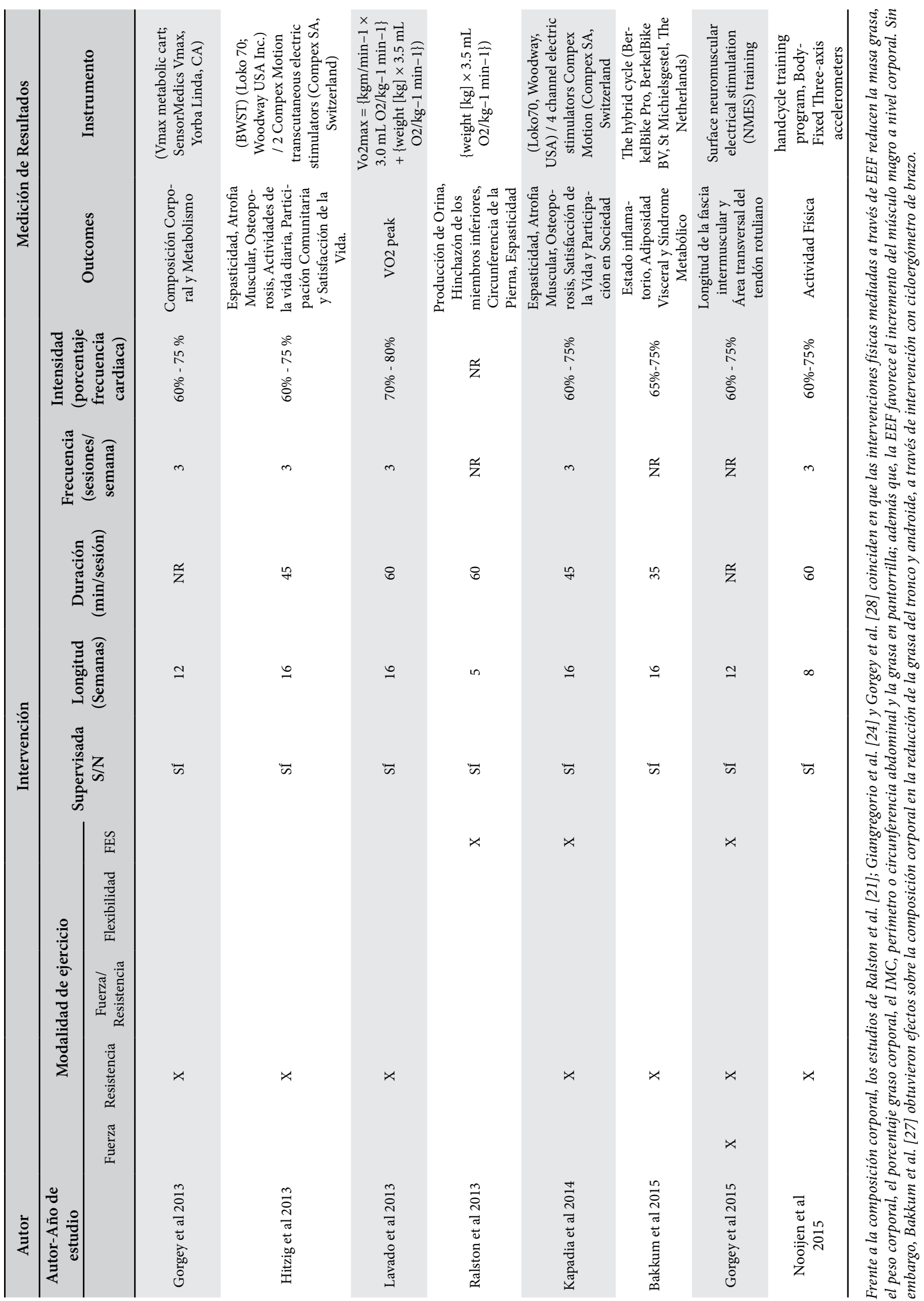


Los ECCA [7, 21, 23, 24] a diferencia de Harvey et al. [19]; Ralston et al. [21]; Nooijen et al. [22]; Kapadia et al. [26]; Bakkum et al. [27]; Hicks et al. [29] y Gorgey et al. [30] reportaron presencia de efectos adversos en las intervenciones mediadas a través de AF y EF, dentro de ellos se destacan: aumento del dolor del cuello y abrasión del codo, pérdida de la conciencia o desmayo, rodillas hinchadas, dolor en el codo izquierdo, sonido en los oídos y mareo, úlcera de presión que requiere hospitalización, infección renal que requiere hospitalización, cirugía de la vejiga, abandonos del estudio secundarios a problemas médicos y abandonos del estudio debido a la asignación al azar.

Por otra parte, el tratamiento convencional para estos pacientes tradicionalmente sugiere intervenciones de tipo quirúrgicas y farmacológicas, siendo el EF considerado un tratamiento complementario, que demanda mayor cuerpo de investigación, ya que sus efectos sobre los componentes de la salud física no son suficientemente conocidos [36, 37]. Entre las características que no permiten generalizar los resultados de los estudios analizados, están: el pequeño tamaño de muestra utilizado en algunos estudios [19, 27, 30], la heterogeneidad en los procesos de evaluación, tanto de fuerza, resistencia muscular, rendimiento funcional, composición corporal y capacidad física y el no control de algunas variables de confusión, como el uso de medicamentos, estado nutricional y niveles de actividad física $[23,24,38]$.

Se sugiere continuar con estudios observacionales o experimentales, controlando las variables confusoras anteriormente descritas, para poder evidenciar el potencial impacto del EF sobre los componentes de la salud física. Lo anterior, le permitirá al profesional de la salud o del ejercicio físico considerar el EF como un criterio pronóstico de la calidad de vida o una herramienta de tratamiento para atenuar los efectos del tratamiento convencional en pacientes con LM.

\section{AGRADECIMIENTOS}

Agradecemos a José Meneses Echávez quien impulsó, en primera instancia, la elaboración de este proyecto y a la médica y cirujana Paola Andrea Muñoz Pérez, quien facilitó sus conocimientos profesionales para esclarecer el proceso fisiopatológico de la LM.

\section{REFERENCIAS}

[1] Lema H, Pérez C, Parra J. Modelo predictivo del grado de discapacidad en adultos con lesión medular: Resultados desde el Who-Das II. Ciencias de la Salud. 2011 May; 2(48): 159-172. http://www.scielo.org.co/scielo. php?script=sci_arttext\&pid=S1692-72732011000200005

[2] Wyndaele C, Wyndaele J. Incident, prevalence, and epidemiology of spinal cord injury: What learns a worldwide literature survery? Spinal Cord. 2006 Feb; 23(10): 523-529. DOI: https://doi.org/10.1038/sj.sc.3101893

[3] Sisto A, Evans S, Nick. Activity and fitness in spinal cord injury: review and update. Curriculum Physical Medical Rehabilitation. 2014 Jun; 3(3): 147-157. DOI: https://doi.org/10.1007/s40141-014-0057-y

[4] OMS. World Health Organization. [Online]; 2013 [Update 2016 august 26; cited 2014 Abril 10]. Available from: http://www.who.int/mediacentre/factsheetd/fs384/es/.

[5] Froelich-Grobe K, Lee J, Aaronson L, Nary D, Washburn R, Little T. Exercise for Everyone: A Randomized Controlled Trial of Project Workout on Wheels in Promoting Exercise Among Wheelchair Users. Archives of Physical Medicine and Rehabilitation. 2014 Aug; 20(95): 20-28. DOI: https://doi.org/10.1016/j.apmr.2013.07.006

[6] Arsom K, Ginis M, Jorgensen S, Stapleton J. Exercise and Sport for Persons Whit Spinal Cord Injury. Physical Medicine and Rehabilitation. 2012 Apr; 27(84): 894-900. DOI: https://doi.org/10.1016/j.pmrj.2012.08.006

[7] Harvey LA, Lin CW, Glinsky J, De Wolf A. The Effectiveness of Physical Interventions for People whit Spinal Cord Injuries: A Systematic Review. Spinal Cord. 2009 Jun; 47(71): 184195. DOI: https://doi.org/10.1038/sc.2008.100

[8] Kathleen A, Arbour-Nicitopuolus K, Ginis M, A.E L. Planning leisure-time physical activity and coping self-efficacy in persons whit spinal cord Injury: A randomized controlled trial. Archives of Physical Medicine and Rehabilitation. 2009 Dec; 90(19): 20032011. DOI: https://doi.org/10.1016/j.apmr.2009.06.019

[9] Hicks AL, Ginins M, Pelletiere C, Ditor D, Foulon B, Wolfe D. The Development of Evidence-Informed Physical Activity Guidelines for Adults whit Spinal Cord Injury: A Systematic Review. Spinal Cord. 2011 Jun; 49(11): 1088-1096. DOI: https://doi.org/10.1038/sc.2011.63

[10] Swinnen E, Duerinck S, Baeyens JP, Meeusen R, Kerckhfs E. Effectiveness of robot-assisted gait training in pesons with spinal cord injury: A systematic review. Journal of Rehabilitation Medicine. 2010 Enero; 42(6): 520-526. DOI: https://doi.org/10.2340/16501977-0538

[11] Liberati A, Douglas GA, Jennifer T, Cynthia M, Gotzche P, John PAI, et al. The PRISMA statement for reporting 
systematic reviews and meta-analyses of studies that evaluate health care Interventions: explanation and elaboration. PLoS Medicine, BMJ, Journal of Clinical Epidemiology, and Open Medicine. 2009 Julio; 62(10): e1-e34. DOI: https://doi.org/10.1016/j.jclinepi.2009.06.006

[12] Moher D, Liberati A, Jennifer T, Altman D, Group atP. Reprint preferred reporting items for systematic reviews and meta-analyses: the PRISMA statement. PLoS Medicine, BMJ, Journal of Clinical Edidemiology, and Open Medicine. 2009 Septiembre; 89(9): 873-880. DOI: https://doi.org/10.1093/ptj/89.9.873

[13] Higgins J, Green S. Cochrane collab. [Online]. 2011 [Update 2016 september 17; cited 2017 Abril 10. Available from: www.handbook.cochrane.org.

[14] Robinson K, Dickersin K. Development of a higlhy sensitive search strategy for the retrieval of reports of controlled trials using PubMed. International Journal of Epidemiology. 2002 Octubre; 31(1): 150-156. DOI: https://doi.org/10.1093/ije/31.1.150

[15] McNeely M, Campbell K, Rowe B, Klassen T, Mackey J, Courneya K. Effects of exercise on breast cancer patients and survivors: a systematic review and meta-analysis. Clinical Medicine Archives Journal. 2006 Julio; 175(1): 34-41. DOI: https://doi.org/10.1503/cmaj.051073

[16] Association AP. PEDro Physiotherapy evidence database. [Online]; 2017 [Update january 19; cited 2017 Septiembre 24. Available from: https://www.pedro.org.au/.

[17] Verhagen A, de Vet H, de Bie R, Kessels A, Boers M, Bouter $\mathrm{L}$, et al. The delphi list: A criteria List for quality assessment of randomized clinical trials for conducting systematic reviews developed by delphi consensus. Journal Clinical of Epidemiology. 1998 Febrero; 51(12): 1235-1241. DOI: https://doi.org/10.1016/S0895-4356(98)00131-0

[18] Moseley A, Herbert R, Sherrington C, Mher C. Evidence for physiotherapy practice: A survey of the physiotherapy evidence database (PEDro). Australian Journal of Physiotherapy. 2002 Abril; 48(1): 43-49. DOI: https://doi. org/10.1016/S0004-9514(14)60281-6

[19] Harvey L, Fornusek C, Bowden J, Pontifex N, Glinsky J, Middleton J, et al. Electrical stimulation plus progressive resistance training for leg strength in spinal cord injury: A randomized controlled trial. Spinal Cord. 2010 Enero; 48(7): 570-575. DOI: https://doi.org/10.1038/sc.2009.191

[20] Lavado E, Cardoso J, Silva L, Dela Bela L, Atallah A. Effectiveness of aerobic physical training for treatment of chronic asymptomatic bacteriuria in subjects with spinal cord injury: a randomized controlled trial. Clinical Rehabilitation. 2013 Enero; 27(2): 142-149. DOI: https://doi.org/10.1177/0269215512450522

[21] Ralston K, Harvey L, Batty J, Lee B, Ben M, Cusmiani R et al. Functional electrical stimulation cycling has no clear effect on urine output, lower limb swelling, and spasticity in people with spinal cord injury: a randomised cross-over trial. Journal of Physiotherapy. 2013 junio; 59(4): 237-243. DOI: https://doi.org/10.1016/S1836-9553(13)70200-5

[22] Nooijen C, Stam H, Bergem M, Bongers-Janssen H, Valent L, van Lageveld S, et al. A behaivioural intervention increase physical activity in people with subacute spinal cord injjury: a randomised trial. Journal of Physiotherapy. 2016 Enero; 62(1): 35-41. DOI: https://doi.org/10.1016/j.jphys.2015.11.003

[23] Mulroy S, Thompson L, Kemp B, Hatchett P, Newsan C, Gutierrezz Lupold D, et al. Strengthening and optimal movements for painful shoulders (STOMPS) in chronic spinal cord injury: A randomized controlled trial. Physical Therapy Clinical Research Network. 2011 Marzo; 91(3): 305-324. DOI: https://doi.org/10.2522/ptj.20100182

[24] Giangregorio L, Craven C, Richards K, Kapadia N, Hitzig S, Masani K, et al. A randomized trial of functional electrical stimulation for walking in incomplete spinal cord injury: Effects on body composition. Jouranl of Sppinal Cord Medicine. 2012 october; 35(5): 351-360. DOI: https://doi.org/10.1179/2045772312Y.0000000041

[25] Hitzig S, Craven C, Panjwani A, Kapadia N, Giangregorio L, Richards K, et al. Randomized trial of functional electrical stimulation therapy for walking in incomplete spinal cord injury: effects on quality of life and community participation. Spinal Cord Injury Rehabilitation. 2013 march; 19(4): 245-258. DOI: https://doi.org/10.1310/sci1904-245

[26] Kapadia N, Masani K, Craven C, Giangregorio L, Hitzig $\mathrm{S}$, Richards $\mathrm{K}$, et al. A randomized trial of functional electrical stimulation for walking in incomplete spinal cord injury: Effects on walking competency. Journal of Spinal Cord Medicine. 2014 april; 37(5): 511-524. DOI: https://doi.org/10.1179/2045772314Y.0000000263

[27] Bakkum A, Paulson T, Bishop N, Goosey-Tolfrey V, Stolwijk-Swuste J, van Kuppelvelt D, et al. Effects of hybrid cycle and handcycle excercise on cardiovascular disease risk factor in people with spinal cord injury: A randomized controlled trial. Journal of Rehabilitation Medicine. 2015 may; 47(6): 523530. DOI: https://doi.org/10.2340 /16501977-1946

[28] Gorgey A, Mather K, Cupp H, Gater D. Effects of resistance training on adiposity and metabolism after spinal cord injury. Journal of the American College of Sports Medicine. 2012 august; 44(1): 165-174. DOI: https://doi.org/10.1249 / mss.0b013e31822672aa

[29] Hicks A, Martin K, Ditor D, Latimer A, Craven C, Bugaresti J, et al. Long-term exercise training in persons with spinal cord injury: efects on streng- 
th, arm ergometry performance and psychological well-being. Spinal Cord. 2003 may; 41(1): 34-43. DOI: https://doi.org/10.1038/sj.sc.3101389

[30] Gorgey A, Khalil R. Neuromuscular electrical stimulation training increases intermuscular fascial length but not tendon cross-sectional area after spinal cord injury. Spinal Cord Injury Rehabilitation. 2015 june; 21(1): 8792. doi: 10.1310/sci2101-87.

[31] Nash M. S, van de Ven I, van Elk N, Johnson B. M. Resistance training program of the upper extremity in manual wheelchair users. Archives of Physical Medicine and Rehabilitation. 2007 january; 88(1): 70-75. DOI: https://doi.org/10.1016/j.apmr.2006.10.003

[32] Ditor D, Latimer A, Martin Ginins K, Arbour K, McCartney N, Hicks A. Maintenance of exercise participation in individuals with spinal cord injury: effects on quality of life, stress, and pain. Spinal Cord. 2003 november; 41(8): 446-450. DOI: https://doi.org/10.1038/sj.sc.3101487

[33] Bakkum A, de Groot S, van der Woude L, Janssen T. The effects of hybrid cycle training in inactive people with long-term spinal cord injury: design of a multicenter randomized controlled trial. Disability and Rehabilitation. 2012 june; 35(13): 1127-1132. DOI: https://doi.org/10.3109/09638288.2012.715719

[34] Wirz M, Zemon D, Rupp R, Schell A, Colomo G, Dietz $\mathrm{V}$. Effectiveness of automated locomotor trainning in patients with chronic incomplete spinal cord injury: a multicenter trial. Archives of Physiscal Medicine and Rehabilitation. 2005 august; 86(4): 672-680. DOI: https://doi.org/10.1016/j.apmr.2004.08.004

[35] Wessels M, Cees L, Inge E, de Groot S. Body-weight-supported gait training for restoration of walking in people with an incomplete spinal cord injury: A systematic review. Journal of Rehabilitation Medicine. 2010 january; 42(6): 513-519. DOI: https://doi.org/10.2340 /16501977-0525

[36] Jiménez B, Martín J, Abadía O, Herrero JA. Resistance Training Program of the Upper Extremity in Manual Wheelchairs Users. Archives of Physiscal Medicine and Rehabilitation. 2007 september; 7(27): 152-159. DOI: https://doi.org/10.1016/j.apmr.2006.10.003

[37] Harvey L, Herbert R, Glinsky J, Moseley A, J B. Effects of 6 months of regular passive movements on ankle joint mobility in people with spinal cord injury: a randomized controlled trial. Spinal Cord. 2009 june; 47(1): 62-66. DOI: https://doi.org/10.1038/sc.2008.71

[38] Hicks A. L, Ginis K. M, Pelletier C. A, Ditor D. S, Foulon B, Wolfe D. L. The effects of exercise training on physical capacity, strength, body composition and functional performance among adults with spinal cord injury: a systematic review. Spinal Cord. 2011 june; 49(11): 11031127. DOI: https://doi.org/10.1038/sc.2011.62

\section{Correo electrónico de los autores:}

Daniel Efrén García-González: danielegg05@gmail.com

Carolina Sandoval-Cuellar: carolinasandovalmaestria@gmail.com 